\title{
Staphylococcus lugdunensis Endocarditis in a Patient Being Treated for Pyelonephritis
}

\author{
Christopher M Peake ${ }^{1}$, Nicholas Faure-Walker ${ }^{2}$, Elli DeMertzi ${ }^{2}$ \\ ${ }^{1}$ St George's University Hospital, Blackshaw Road, Tooting, London, UK \\ ${ }^{2}$ Kingston Hospital, Galsworthy Road, Kingston-upon-Thames, UK
}

\begin{abstract}
A 35 years old female with known chronic left pelvo-ureteric junction (PUJ) obstruction presented with recurrent pyrexia and left loin pain and was treated for pyelonephritis. She had a history of severe aortic valve (AV) regurgitation. After five days of broad spectrum antibiotics and a percutaneous nephrostomy, her fevers persisted. Staphylococcus lugdunensis was isolated from her blood culture and she was diagnosed with mitral valve endocarditis following an echocardiogram. She was transferred to a tertiary cardiothoracic unit where she underwent a mitral and aortic mechanical valve repair. After 6 weeks of intravenous antibiotic treatment she was discharged without signs of infection but with poor cardiac function. S. lugdunensis is a virulent organism which is thought to be underdiagnosed and is associated with aggressive and destructive endocarditis. An awareness of the organism as well as prompt diagnosis and treatment with effective multi-disciplinary management is needed to avoid substantial morbidity in these patients.
\end{abstract}

Whilst $S$. lugdunensis is known to cause invasive endocarditis, it has never been reported following pyelonephritis. The significance of this case is that despite receiving appropriate and timely treatment the patient was left with deteriorating cardiac function. This is an important reminder that this organism must be taken seriously when isolated in the context of infection. J Microbiol Infect Dis 2018; 8(1):37-40

Keywords: Staphylococcus lugdunensis, endocarditis, pyelonephritis, heart valves, urinary tract

\section{INTRODUCTION}

Staphylococcus lugdunensis is a coagulasenegative Staphylococcus (CoNS) first identified in 1988 in Lyon, France. S. lugdunensis can be a normal skin commensal but can also cause severe, life threatening disease. In its pathogenic state, it can cause virulent infections similar to those associated with staphylococcus aureus. It is part of the normal skin flora with preponderance for the groin, axilla and toes [1]. It is associated with cardiovascular, prosthetic joint, bloodstream, skin and soft tissue, nervous system and urinary tract infections as well as peritonitis, endopthalmitis and osteomyelitis [2]. It has been described in severe native and prosthetic valve and device-related endocarditis, myocarditis and infected myxoma [3]. Native valve endocarditis is commonly associated with the mitral valve with mortality reported as high as $42 \%$ [4]. Native valve infection has been reported after vasectomy $[5,6]$ and renal transplant [7]. Most isolates of S. lugdunensis have been found to be susceptible to methicillin as well as a wide range of antimicrobial agents.

\section{CASE REPORT}

A 35-year old Polish lady presented for the third time in seven weeks with left loin pain, fever and rigors associated dysuria and urinary frequency. She was known to have a chronic left pelvicoureteric junction (PUJ) obstruction with 39\% function in the left kidney on a MAG3 urogram. Her PUJ obstruction had been managed with ureteric stents and she was awaiting a robotic pyeloplasty which had been delayed owing to recurrent episodes of pyelonephritis. The last stent was removed seven months before as the kidney was draining well. On previous presentations with pyelonephritis, urine cultures had isolated Escherichia coli fully sensitive to all antibiotics tested. She was treated with oral 
ciprofloxacin and intravenous (IV) gentamicin followed by meropenem and ertapenem.

She was also known to have a bicuspid aortic valve, aortic regurgitation (AR) and mild left ventricular dilatation. She suffered from panic attacks and did not take any regular medications. She was allergic to penicillin. She was a smoker and drunken approximately 4 units of alcohol per week. She had never injected any recreational drugs.

On presentation she had temperature of $38.8^{\circ} \mathrm{C}$, heart rate of $125 \mathrm{bpm}$ with white cell count (WCC) of $13.3 \times 10^{9} / \mathrm{L}$ and C-reactive protein (CRP) of $177 \mathrm{mg} / \mathrm{L}$, normal renal function. An ultrasound scan of the renal tract showed severe left hydronephrosis consistent with chronic PUJ obstruction.

She was initially treated with IV meropenem, fluids and analgesia. After two days, she had hypotensive episodes and her fevers persisted. There was no biochemical improvement. The interventional radiologists inserted a percutanous nephrostomy tube which drained clear urine. One day later, from the blood culture taken on admission Staphylococcus lugdunensis was isolated. It was sensitive to penicillin, flucloxacillin, erythromycin, clindamycin, fucidic acid, rifampicin, tetracycline, gentamicin, ciprofloxacin, trimethoprime, teicoplanin and vancomycin. The identification of the isolate was made by Maldi-Tof (mass spectrometry) and sensitivity testing by disks diffusion (BSAC) and E-tests. Following microbiology advice, the patient started IV teicoplanin. The nephrostomy fluid did not culture any bacteria. A further blood culture taken the second day of admission also confirmed S. lugdunensis and IV teicolpanin was changed to vancomycin.

The patient continued to spike fevers up to 39.3 ${ }^{0} \mathrm{C}$. Her CRP and WCC remained high at 197 $\mathrm{mg} / \mathrm{L}$ and $15.1 \times 10^{9} / \mathrm{L}$ respectively. An MRI of the abdomen and spine did not identify any other sources of infection. On day 5, a transthoracic echocardiogram identified a large mass adherent to the mitral valve (MV) anterior leaflet at the junction between the aortic valve (AV) and $M V$ with Moderate MV obstruction and persistent severe AR. She was reviewed by the Cardiology team who identified a systolic murmur, splinter hemorrhages, a high volume pulse but no Roth spots or Janeway lesions. The patient was diagnosed with a MV endocarditis and transferred urgently to a tertiary cardiothoracic surgery center. Rifampicin was added on seventh day. She underwent a trans-esophageal echocardiogram (TOE) on ninth day which showed a frond like vegetation on the anterior MV leaflet $(26 \mathrm{~mm})$, mild mitral regurgitation, an aortic root abscess with destruction of the aortic valve and severe regurgitation.

In terms of the modified Dukes criteria for diagnosing infective endocarditis, she had an echocardiogram showing a mass on the valve leaflet and abscess (major criteria). She also had a pre-existing bicuspid aortic valve with AR, a temperature of above $38.0{ }^{\circ} \mathrm{C}$ and a positive blood culture for $S$. lugdunensis from two separate cultures in the context of active infection (three minor criteria). Although she had splinter haemorrhages on clinical examination, these are not included within the criteria $[8,9]$.

The patient went on to have debridement of the abscess cavity, mitral and aortic valves on tenth day. Operative findings were consistent with echocardiogram with large vegetation found on the anterior leaflet of the MV, destruction of the AV and a large abscess cavity at the annulus. The MV was replaced with a $27 \mathrm{~mm}$ St Jude mechanical valve and the AV with a $19 \mathrm{~mm}$ Medtronic ATS mechanical valve. The abscess cavity was closed. Intra-operative samples cultured did not grow any organisms after prolonged incubation.

After completing 6 weeks of intra-venous antibiotics she was discharged with no signs of infection. A post-operative echocardiogram showed well seated mechanical atrial and mitral valves with evidence of redundant cardiac muscle in the LV cavity and an ejection fraction of $35-45 \%$.

\section{DISCUSSION}

This case highlights the difficulty in diagnosing and management of $S$. lugdunensis infection as well as the complications that can result. This organism shares many of its virulence factors with Staphylococcus aureus, with similar pathogenic potential. Shared virulence factors include the ability to escape phagosome action through production of gamma-like haemolysin [10], lysozyme resistance due to a homologous OatA region of the genome [11] and binding to key factors in cell adhesion and wound healing 
[2]. It can also cause a toxic shock syndrome [12] and produce a biofilm [13].

There is a paucity of cases describing $S$. lugdunensis endocarditis in the literature and general lack of awareness of the organism [14]. Despite this, it is known as a very destructive cause of endocarditis that cannot be missed $[2,14]$. It carries with it a very high rate of morbidity and mortality even with appropriate treatment as described by this case. Despite this, there is still a lack of randomized control data on the efficacy of antimicrobial agents. However there have been suggestions that early surgical intervention with native valve endocarditis results in good outcomes [5].

$S$. lugdunensis is rarely a contaminant but there is evidence to suggest that there is diagnostic difficulty in determining pathological from contaminate strains. As described by E. Babu et al, its similarities to $S$. aureus as well as the 'clumping factor' has led to the two organisms being confused during latex agglutination testing [15]. The organism could be incorrectly identified as a mixed population, as found in contaminate samples, due to its colony variation [16,2]. Further to this, some automated identification systems fail to identify $S$. lugdunensis depending on the database and testing used $[17,18]$.

In this case, neither the cultures of the urine, nor the intra-operative vegetation samples grew $S$. lugdunensis. However, the clinical course, investigations and repeated growth of the organism in blood cultures provided the strong suspicion that the organism was the cause of both the initial pyelonephritis and subsequent endocarditis. Several case reports and series have described $S$. lugdunensis infective endocarditis originating from the urinary tract. Haile et al. has suggested that $S$. lugdunensis may be an unrecognized yet infrequent cause of urinary tract infections [19].

It is clear that this organism has high virulence potential and can result in severe infections. Despite being rare, it is likely underdiagnosed, difficult to treat and has been identified in several cases where the origin was the urinary tract. With this in mind, an awareness of the organism as well as prompt diagnosis and effective multi-disciplinary management is needed to avoid substantial morbidity in these patients.

\section{CONCLUSION}

This case demonstrates how S. lugdunensis, presenting as a pyelonephritis and likely originating from the urinary tract, caused an aggressive form of native valve infective endocarditis in a young woman with pre-existing cardiac pathology. It was expedient consultation with microbiology specialists and a multidisciplinary approach that lead to appropriate investigation and subsequent treatment strategy. Despite optimum management the patient was left with poor and deteriorating cardiac function.

S. lugdunensis endocarditis has not been associated with pyelonephritis before in published literature and there is still a lack of general awareness about the organism amongst clinicians. This case should therefore act to, not only raise awareness of the organism but also act as a warning to clinicians who come across it in their clinical practice.

\section{ACKNOWLEDGMENTS}

Conflict of interest: The authors declare no personal or financial conflict of interest.

Financial Disclosure: No financial support was received

Consent: Written informed consent was obtained from the patient for publication of this case report.

\section{REFERENCES}

1.Beiber L, Kahlmeter G. Staphylococcus lugdunensis in several niches in the normal skin flora. Clin Microbiol Infect 2010; 16(4): 385-388.

2.Babu E, Orpello J. Staphylococcus lugdunensis: the coagulase negative staphylococcus you don't want to ignore. Expert Review of Anti-Infective Therapy 2011; 9(10): 901-907.

3.Bhanot N, Sahud AG, Bhat S, et al. Fever of unknown origin: a case of cardiac myxoma infected with Staphylococcus lugdunensis. South Med J 2010; 103(7): 697-700.

4.Anguera I, Del Río A, Miró JM, et al. Staphylococcus lugdunensis infective endocarditis: description of 10 cases and analysis of native valve, prosthetic valve, and pacemaker lead endocarditis clinical profiles. Heart 2005; 91(2): e10.

5.Fervenza FC, Contreras GE, Garratt KN, et al. Staphylococcus lugdunesis endocarditis: a complication of vasectomy? Mayo Clinic Proc 1999; 74(12): 1227-1230.

6.Schandiz H, Olav Hermansen N, Jørgensen T, et al. Staphylococcus lugdunensis endocarditis following vasectomy - a report of a case history and review of literature. APMIS 2015; 123: 726-729.

7.Patel R, Piper KE, Rouse MS, et al. Frequency of isolation of Staphylococcus lugdunensis among 
staphylococcus isolates causing endocarditis: a 20year experience. J Clin Micorbiol 2000; 38(11): 4262426.

8.Durack DT, Lukes AS, Bright DK. New criteria for diagnosis of infective endocarditis: utilization of specific echocardiographic findings. Am J Med 1994; 96(3): 200-9

9.Li JS, Sexton DJ, Mick N, Nettling $R$, et al. Proposed modification to the Duke criteria for diagnosis of infective endocarditis. Clin Infect Dis 2000; 30(4): 633-8

10.Giese B, Glowinski F, Paprotka K, et al. Expression of $\delta$-toxin by Staphylococcus aureus mediated escape from phago-endosomes of human epithelial and endothelial cells in the presence of $\beta$ toxin. Cell Microbiol 2011; 13(2): 316-329.

11.Bera A, Biswas R, Herbert $S$, et al. The presence of peptidoglycan O-acetyltranferase in various staphylococcal species correlates with lysozyme resistance and pathogenicity. Infect Immun 2006; 74(8): 4598-4604.

12.Pareja J, Gupta K, Koziel H. The toxic shock syndrome and Staphylococcus lugdunensis bacteremia. Ann Intern Med 1998; 128(7): 603-604.

13.Von Eiff C, Peters G, Heilmann C. Pathogenesis of infections due to coagulase-negative staphylococci. Lancet Infect Dis 2002; 2(11): 677-685.

14. Liu P, Huang Y, Tang C. Staphylococcus lugdunensis Infective Endocarditis: A literature review and analysis of risk factors. J Micro, Immun Infection 2010; 43(6): 478-84.

15.Paulsson M, Petersson AC, Ljungh A. Serum and tissue protein binding and cell surface properties of Staphylococcus lugdunensis. J Med Microbiol 1993; 38(2): 96-102.

16.Arciola CR, Campoccia D, An YH, et al. Prevalence and antibiotic resistance of 15 minor staphylococcal species colonizing orthopaedic implants. Int J Artif Organs 2006; 29(4): 395-401.

17.Grant CE, Sewell DL, Pfaller M, et al. Evaluation of two commercial systems for identification of coagulase-negative staphylococci to species level. Diagn Microbiol Infect Dis 1994; 18(1): 1-5.

18.Kloos WE, George CD. Identification of Staphylococcus species and subspecies with the MicroScan Pos ID and Rapid Pos ID panel systems. J Clin Microbiol 1991; 29(4): 738-744.

19. Haile DT, Hughes J, Vetter E, et al. Frequency of isolation of Staphylococcus lugdunensis in conservative urine cultures and relationship to urinary tract infection. J Clin Microbiol 2002; 40(2): 654-656. 